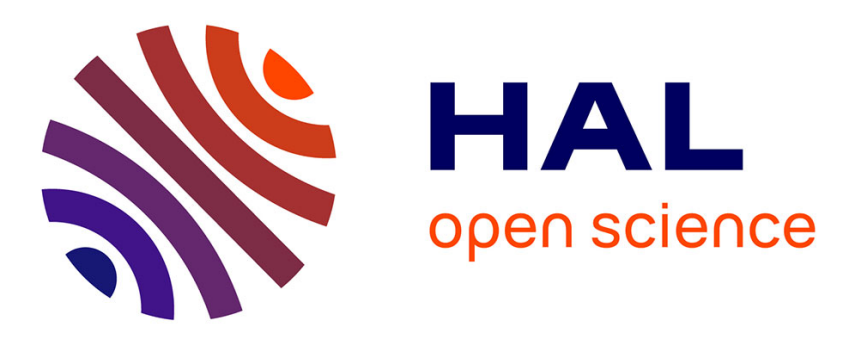

\title{
Performance of VAMOS for reactions near the Coulomb barrier
}

\author{
S. Pullanhiotan, M. Rejmund, A. Navin, W. Mittig, S. Bhattacharyya
}

\section{To cite this version:}

S. Pullanhiotan, M. Rejmund, A. Navin, W. Mittig, S. Bhattacharyya. Performance of VAMOS for reactions near the Coulomb barrier. Nuclear Instruments and Methods in Physics Research Section A: Accelerators, Spectrometers, Detectors and Associated Equipment, 2008, 593, pp.343-352. 10.1016/j.nima.2008.05.003 . in2p3-00288214

HAL Id: in2p3-00288214 https://hal.in2p3.fr/in2p3-00288214

Submitted on 27 Jun 2008

HAL is a multi-disciplinary open access archive for the deposit and dissemination of scientific research documents, whether they are published or not. The documents may come from teaching and research institutions in France or abroad, or from public or private research centers.
L'archive ouverte pluridisciplinaire $\mathbf{H A L}$, est destinée au dépôt et à la diffusion de documents scientifiques de niveau recherche, publiés ou non, émanant des établissements d'enseignement et de recherche français ou étrangers, des laboratoires publics ou privés. 


\title{
Performance of VAMOS for reactions near the Coulomb barrier
}

\author{
S.Pullanhiotan, ${ }^{1}$ M. Rejmund, A. Navin, W. Mittig, S. Bhattacharyya ${ }^{2}$ \\ Grand Accélérateur National d'Ions Lourds (GANIL), CEA/DSM - CNRS/IN2P3, Bd Henri Becquerel, BP 55027, F-14076 Caen, France.
}

\begin{abstract}
VAMOS (VAriable MOde Spectrometer) is a large solid angle ray-tracing spectrometer employing numerical methods for reconstructing the particle trajectory. Complete identification of reaction products has been achieved by trajectory reconstruction. Equipped with a versatile detection system, VAMOS is capable of identifying reaction products from diverse reactions using beams at GANIL. The technique for trajectory reconstruction and its application for identifying reaction products are presented. The angular acceptance of the spectrometer has been studied using Monte Carlo simulation by an ion optics code. The spectrometer was coupled to the high efficiency EXOGAM $\gamma$-array to obtain $\gamma$-recoil coincidences for studying nuclei far from stability. The main features of the spectrometer as well as some results applied to experiments in deep inelastic collisions are described.
\end{abstract}

Key words: Magnetic spectrometer, trajectory reconstruction, Particle Identification, Nuclear structure PACS: 07.55.-w, 25.70.-z, 29.30.-h

\section{Introduction}

In recent years, new generation of magnetic spectrometers $[1,2,3]$ are playing crucial role in the study of nuclei far from stability. These spectrometers classified as "raytracing spectrometers" have large solid angle and momentum acceptance well suited for reactions with stable as well as radioactive ion beams. The VAMOS spectrometer (VAriable MOde Spectrometer) [1] operating at GANIL is one such large acceptance ray-tracing spectrometer, now being extensively used for the identification of reaction products in heavy ion collisions produced with both stable and radioactive ion beams from ISOL [4] and fragmentation facility at GANIL. The spectrometer has been designed to cover a wide range of reactions such as elastic and inelastic scattering, transfer, deep inelastic and fusion evaporation reactions utilizing both the conventional and inverse kinematics. In inverse reactions (heavy projectile directed onto light target) the recoils are kinematically focused in the forward direction with a higher energy, which aids in improving their collection and detection efficiency. The nature and kinematics of these diverse reactions demand a high perfor-

\footnotetext{
1 Permanent Address: Inter University Accelerator Centre, Aruna Asaf Ali Marg, New Delhi, 110067, India and Dept. of Nuclear Physics, Andhra University, Visakhapatnam-530003,Andhra Pradesh, India.

2 Permanent Address: Variable Energy Cyclotron Centre, 1/AF Bidhan Nagar, Kolkata 700064, India.
}

mance device that accepts reaction products at large angles and detect them efficiently at all range of accepted energies. The energy of the reaction products depends on the kinematics and the detection system must be efficient and sensitive to both slow moving heavy recoils and fast light particles. To meet these requirements, the VAMOS spectrometer was built based on a unique design that combines different modes of operation in a single device. Depending upon the kinematics of the reaction, the operating mode of VAMOS can be varied to optimize different experimental requirements.

In the momentum dispersive mode of operation, the spectrometer selects and separates the reaction products at the focal plane according to their momentum to charge $(\mathrm{p} / \mathrm{q})$ ratio and their unique identification is achieved via an event-by-event reconstruction of ion trajectory in the magnetic fields. This eliminates the need for a position sensitive detector (near the target) to reconstruct the scattering angle and thus allows the spectrometer to be used at $0^{\circ}$. When operating as a velocity filter at zero degrees, VAMOS physically separates the reaction products from direct beam and transports them to the focal plane. This flexibility combined with its large acceptance and versatile detection system makes the spectrometer a unique device to separate and uniquely identify the reaction products in terms of A (mass number) and $\mathrm{Z}$ (proton number). The spectrometer when coupled with the high efficiency $\gamma$ detector array EXOGAM [5] provides a powerful tool for the 


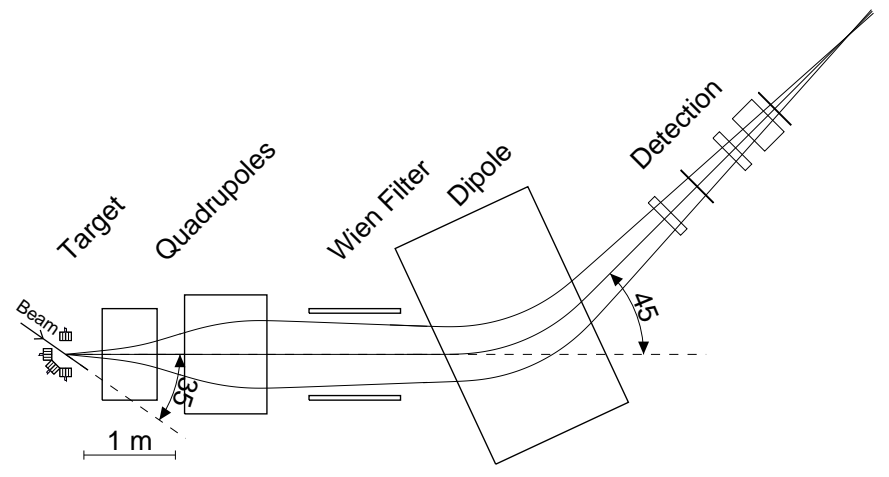

Figure 1. Schematic layout of VAMOS Spectrometer showing the ion optical elements. The spectrometer is shown rotated to $35^{\circ}$ with respect to beam direction and the detector system placed at an angle of $45^{\circ}$.

spectroscopy of very weak reaction channels. Using the reconstructed velocity and emission angle at the target, the coincident $\gamma$-rays are Doppler corrected resulting in better resolution of the individual states in final nuclei [6]. In the present manuscript we describe the performance of the VAMOS spectrometer and its detection system for selection and identification of products in inverse kinematics from deep inelastic reactions near the Coulomb barrier.

This paper is organized as follows. In section 2 we describe the general design features of the spectrometer and its specifications. An overview of the focal plane detection system is given in section 3. Section 4 is devoted to the description of trajectory reconstruction, identification of particles produced in deep inelastic transfer collisions and also its application to the Doppler correction for coincident gamma rays. Finally in section 5 we discuss the results on simulation studies on the spectrometer acceptance and compare them with experimental data from quasi-elastic scattering (elastic like).

\section{VAMOS spectrometer at GANIL}

\subsection{Physical Description}

The spectrometer, as shown schematically in Fig. 1, consists of two large aperture magnetic quadrupoles at the entrance, followed by an $E \times B$ Wien filter and a large magnetic dipole. The first quadrupole which focuses in the vertical plane has an opening of $30 \mathrm{~cm}$ diameter and a magnetic length of $60 \mathrm{~cm}$ while the second quadrupole focuses in the horizontal plane(dispersive plane) is elliptical in shape with $100 \mathrm{~cm}$ along the main axis (horizontal) and a magnetic length of $90 \mathrm{~cm}$. In the standard configuration, the first quadrupole is placed at $40 \mathrm{~cm}$ from the target. A unique feature of the spectrometer is that the momentum dispersion at the detection plane can be varied depending on the selected deflection angle (variable from $0^{\circ}$ to $60^{\circ}$ ) of the dipole magnet. The Wien filter is generally used only when the spectrometer operate as a recoil separator for low energy fusion evaporation residues at $0^{\circ}$. The filter has
Table 1

Operational features of VAMOS.

\begin{tabular}{|c|c|}
\hline Horizontal Acceptance & $-125 \mathrm{mrad}$ to $+100 \mathrm{mrad}$ \\
Vertical Acceptance & $\pm 160 \mathrm{mrad}$ \\
Momentum Acceptance & $\pm 5 \%$ (at $25 \mathrm{msr})$ \\
M/q resolution & $\sim 0.6 \%$ \\
Maximum rigidity B $\rho$ & $1.6 \mathrm{~T}-\mathrm{m}$ \\
Deflection Angle $\theta_{\text {dipole }}$ & $0^{\circ}$ to $60^{\circ}$ (Variable) \\
Flight Path length & $\sim 760 \mathrm{~cm}$ \\
Target - Quadrupole distance & 40 to $120 \mathrm{~cm}($ variable) \\
Angular rotation & $0^{\circ}$ to $60^{\circ}$ \\
\hline
\end{tabular}

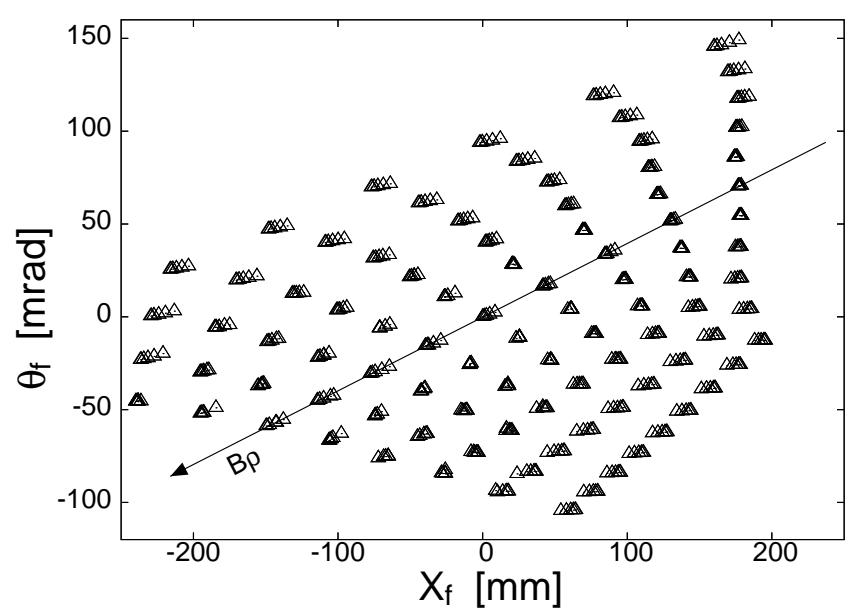

Figure 2. Calculated $(x, \theta)$ image at the image plane of VAMOS showing overall effect of aberrations in position and angle. The figure shows the final positions of the trajectories whose rigidity $B \rho$ and initial angles $\left(\theta_{i}, \phi_{i}\right)$ are varied by $2 \%$ and $20 \mathrm{mrad}$ respectivley. The angles $\left(\theta_{i}, \phi_{i}\right)$ are varied within the range of $\pm 100 \mathrm{mrad}$ and $B \rho$ within $\pm 8 \%$. The line across the figure represents the positions of central rays with $\theta_{i}=0$.

an active volume of $100 \times 100 \times 15 \mathrm{~cm}^{3}$ with maximum electric field of $300 \mathrm{kV}( \pm 150 \mathrm{kV})$ applied across a gap of 100 $\mathrm{cm}$ along the dispersive plane and a maximum magnetic field of 200 Gauss applied across the vertical plane. The focal plane detectors are housed inside a vacuum chamber which can be mounted at different orientations corresponding to various deflection angles of the dipole magnet. The magnets and the detector systems are mounted on two superimposed platforms providing the flexibility of both the rotation and linear motion of the spectrometer. The spectrometer can be rotated around the target from $0^{\circ}$ to $60^{\circ}$. The linear motion allows to increase the distance of the spectrograph from the target $(40 \mathrm{~cm}$ to $120 \mathrm{~cm})$ in order to have more space for installing large ancillary detector arrays around the target. The main operational features of VAMOS spectrometer are summarized in Table 1.

\subsection{Optics for the dispersive mode operation}

In the dispersive mode, VAMOS operates as a traditional QQD magnetic spectrometer in a momentum dispersion 
mode without the use of the Wien filter. The beam optics of the two quadrupoles and magnetic dipole is configured for a point to point focusing of particles at the focal plane keeping the trajectories parallel in the Wien filter region. The optical focal plane of the spectrometer is a tilted plane with an inclination of $85^{\circ}$ to the normal, making it difficult to make any measurement using a single detector along the focal plane. So for all practical purpose, the final coordinates of the particles are determined on an "image plane" which is considered perpendicular to the reference trajectory and is located midway between two tracking detectors. The trajectory coordinates are determined on this image plane by projecting the position and angle measurements obtained from the tracking detectors. When the dipole deflection angle is set at $45^{\circ}$, the dispersion at the image plane is $D \sim 1.8 \mathrm{~cm} / \%$ with a minimum focal plane size of $\sim 40$ $\mathrm{cm}$ length required for a momentum acceptance of $\delta P=$ $\pm 10 \%$. To first order, the length of the focal plane is given by $D \times \delta P$. The first order optics parameters also determine the best possible momentum resolution $R_{p} \sim 10^{-3}$ which is the ultimate limit achievable if all aberrations are corrected or minimized. However, in VAMOS due to its large phase space acceptance, higher order aberrations severely affect the resolution and limit the acceptance significantly. Even though some of the dominant geometrical aberrations are minimized by a careful design of the pole shape of the second quadrupole, with higher order corrections up to fifth order, complete correction of aberrations cannot be achieved by hardware methods alone. The ion optics calculation for VAMOS showed higher order aberrations causing significant image alterations at the focal plane. An example of the overall effect of aberrations in VAMOS can be seen in Fig. 2 which shows the image of a set of trajectories calculated by the ion optics code ZGOUBI [7]. In the figure, the final angle $\theta_{f}$ is plotted as a function of the position $x_{f}$ at the image plane corresponding to the dipole deflection angle of $\theta_{\text {dipole }}=45^{\circ}$. The calculations shown correspond to the trajectories for a set of particles with rigidity $(B \rho)$ and initial angles $\left(\theta_{i}, \phi_{i}\right)$, selected within the range of $\Delta B \rho= \pm 8 \%, \Delta \theta_{i}, \Delta \phi_{i}= \pm 100 \mathrm{mrad}$, and varied by $2 \%$ and $20 \mathrm{mrad}$ respectively. The plot shows trajectories of particles having the same magnetic rigidity but different angles $\left(\theta_{i}, \phi_{i}\right)$ crossing the image plane at different positions. It is evident that a unique determination of $B \rho$ and the scattering angle at the target is not directly possible by a position measurement alone. Hence trajectory reconstruction is an absolute necessity to determine the particle momentum. The description of the trajectory reconstruction and identification method is presented in section 4 .

\section{Detection system for VAMOS}

\subsection{Focal plane detector setup}

Reactions around the Coulomb barrier produce a large number of different nuclei and a unique identification re-

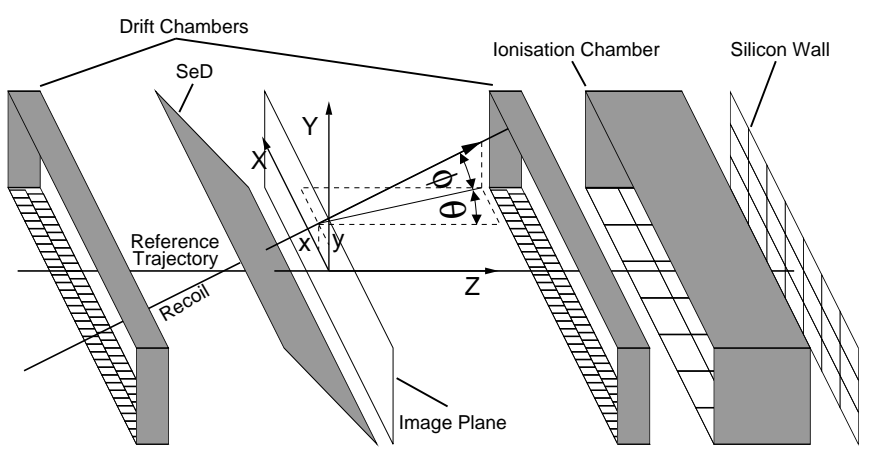

Figure 3. Schematic view of a typical VAMOS focal plane detection setup used in quasi elastic reactions. The coordinate system used in the description of a trajectory is also shown.

quires determination of their mass $M$, atomic number $Z$, charge state $q$, and energy $E$. Additionally, to reconstruct the ion trajectory from the measured final positions and angles, two-dimensional position sensitive tracking detectors with good resolution are required. The VAMOS detection setup uses a combination of detectors at the focal plane measuring position $(x, y)$, energy loss $(\Delta E)$, energy $(E)$ and time of flight $(T O F)$ parameters.

A schematic view of the VAMOS focal plane detection system is shown in Fig. 3. The $x, y$ position coordinates along the trajectory are measured at two planes (perpendicular to the reference trajectory). A pair of identical drift chambers [8] (DC) separated by one meter measures the horizontal $(x)$ and transverse $(y)$ positions of the particles. The drift chambers have discrete pads across the dispersive plane which measure the $x$ coordinate from the positions of the pads on which the induced charges are collected, while the $y$ position is determined from the drift time of electrons reaching the anode wire of the chamber. The measured $x, y$ coordinates at two planes are then used to compute the angles of trajectory and its projection onto an "image plane" located in between the two drift chambers. The computed position and angle coordinates at the image plane are used for trajectory reconstruction on an event-by-event basis. A large area secondary electron detector (SED) [10] mounted behind the first drift chamber is used to obtain a high resolution time (300 ps) reference and trigger signal. The second drift chamber is followed by a $30 \mathrm{~cm}$ deep segmented anode ionization chamber(IC) which measures the differential energy loss $(\Delta E)$ of the particles. An array of silicon detectors mounted behind the ionization chamber is used to stop the particles and provide access to the residual energy $(E)$ and also an additional time reference. The energy loss and residual energy information are used to identify the charge $(Z)$ of the recoils. The time of flight $(T O F)$ is recorded between the beam pulse $(\mathrm{RF})$ and timing from the SED and/or silicon detectors. In experiments where low energy $(E<2 \mathrm{MeV} / \mathrm{u})$ heavy ions are to be detected, the two drift chambers are replaced by two identical SEDs which provide both positions $(x, y)$ and timing information with an effective detector thickness of only $150 \mu \mathrm{gm} / \mathrm{cm}^{2}$ of mylar. 


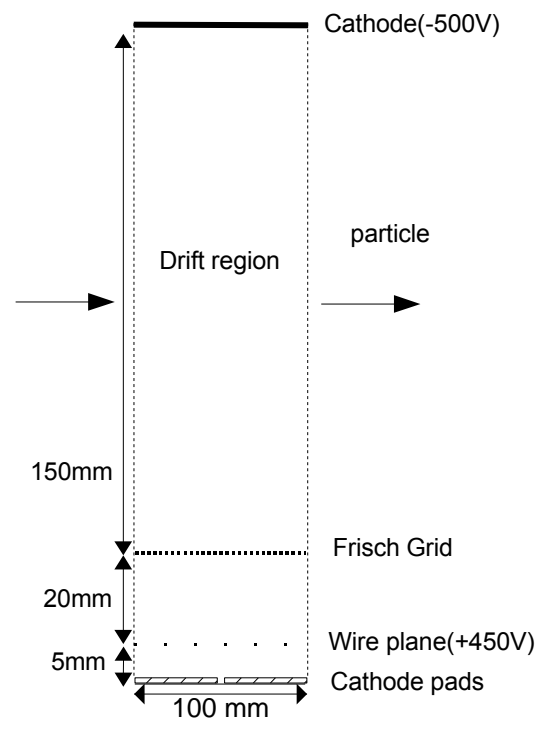

a)

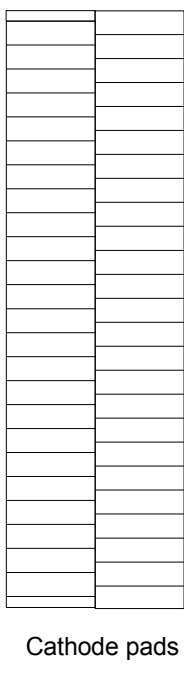

b)
Figure 4. a) Schematic drawing of the drift chamber, b) Segmented cathode showing individual pads. Only a few of the total of 128 pads on two rows are drawn for clarity.

\subsubsection{Drift chambers}

Both drift chambers [8] are of the same size having an active volume of $41 \times 11 \times 10 \mathrm{~cm}^{3}$. A schematic drawing of the drift chamber is shown in Fig. 4. The DC consist of a drift region and an amplification region. The drift region is the active part containing the filling gas where primary ionization occurs. It has a cathode made of a copper plated plane glass epoxy electrode and a Frisch wire grid that separates the drift space from an amplification region. A uniform electric field is created within the drift region using graded potential distribution electrodes along the field direction. The amplification region has a proportional counter consisting of a wire plane electrode fixed between the Frisch grid and a segmented cathode plane. Positive high voltage is applied to the anode wire plane (10 gold plated tungsten wires of $20 \mu \mathrm{m}$ size with $10 \mathrm{~mm}$ spacing, fixed $20 \mathrm{~mm}$ apart from Frisch grid). The Frisch grid is made of $50 \mu \mathrm{m} \mathrm{Cu}$-Be field wires fixed $2 \mathrm{~mm}$ spaced, perpendicular to beam direction. Both the Frisch grid and the segmented cathode are kept at ground potential. The segmented cathode plane consists of two symmetrical planes each further subdivided into 64 separate pads with independent signal readouts. The pads are made of gold plated strips $6 \mathrm{~mm}$ wide at a pitch of 6.5 $\mathrm{mm}$ along the dispersive direction. To reduce the non linearity of the position measurement in between the strips, the two cathode planes are offset by half a strip. All 128 pads are read out individually using GASSIPLEX [9] frontend boards. The detector filled with isobutane gas, is generally operated at 13 mbar pressure with a voltage of $-500 \mathrm{~V}$ on cathode and $+450 \mathrm{~V}$ on the amplification wires respectively. Entrance and exit mylar windows $(0.9 \mu \mathrm{m}$ thick) are used on both sides to isolate the gas from the high vacuum region.

When a particle passes through the DC detector the electrons produced in the ionization process drift towards the amplification wires where they are multiplied and collected. The fast signal from the wire provides the time of arrival of the electrons. At the same time the avalanche induces a signal on nearby cathode pads. A Gaussian fit applied to the charge distribution determines the horizontal $(x)$ position coordinate of the trajectory. The position coordinate $(y)$ in the vertical (non dispersive) plane is determined from the time difference between a reference time $t_{0}$ and the time $t$ taken by the electrons to reach the wires. The reference time $t_{0}$ is provided by the master trigger (timing from SED or Silicon). The $y$ coordinate is determined from the relation $y=v \times\left(t-t_{0}\right)$ where $v$ is the drift velocity of the electrons $(\sim 50 \mathrm{~mm} / \mu \mathrm{s})$ in the gas. The chambers provide a position resolution of 0.3 and $1 \mathrm{~mm}$ (FWHM) for $x, y$ respectively.

\subsubsection{Secondary Electron Detector SED}

The SED detector developed for VAMOS is a large area position sensitive foil detector with a low pressure proportional gas counter as a secondary electron detector [10]. It consists of an aluminized mylar foil of active area $40 \mathrm{x}$ $15 \mathrm{~cm}^{2}$ mounted at $45^{\circ}$ to the particle trajectory. Secondary electrons emitted from the foil are accelerated by a $10 \mathrm{KV}$ potential between the foil and the grid and detected by a gas counter. They are refocused by a parallel magnetic field. The magnetic field is adjusted to have one cyclotron oscillation between the foil and the counter. The signal induced on the position sensing electrodes of the proportional counter are readout individually to localize the avalanche allowing a two dimensional position measurement. The fast timing (300 ps) signal from the detector is used as the master trigger for events at the focal plane.

\subsubsection{Ionization chamber}

The ionization chamber (IC) measures the energy loss of the particles passing through it. It is based on the standard IC design [11] consisting of a segmented anode, Frisch grid and cathode. The anode is divided into two sections along the flight path of the particle. Each section is further segmented into seven pads across the focal plane. The signal from each pad is processed individually by a charge sensitive preamplifier having a typical gain of $20 \mathrm{mV} / \mathrm{pC}$ mounted directly on the detector board. The anode segmentation along the trajectory is fixed at 10 and $20 \mathrm{~cm}$ for the first and second section respectively. The 14 pads on the anode are materialized on a double sided printed circuit boards of $3.2 \mathrm{~mm}$ thickness. The Frisch grid is made of $100 \mu \mathrm{m} \mathrm{Be}-\mathrm{Cu}$ wire of $1 \mathrm{~mm}$ spacing fixed at $15 \mathrm{~cm}$ from the cathode. The cathode is made of a polished metal plate and a uniform electric field is maintained between the Frisch grid and cathode using a series of strip electrodes along each side of the detector. The IC has $1.5 \mu \mathrm{m}$ thick mylar window of size $40 \mathrm{~cm} \mathrm{x} 12 \mathrm{~cm}$ at its entrance while the exit port is directly coupled to the Silicon wall. For particles with higher energy $(E>2 \mathrm{MeV} / \mathrm{u})$ the detector is normally operated with isobutane gas at a pressure of 40 mbar. 


\subsubsection{Silicon Wall.}

At the end of the spectrometer, the particles are fully stopped in an array of Silicon detectors. The array consists of 21 Silicon detectors arranged in $3 \times 7$ matrix covering the active area of the image plane $\left(40 \times 15 \mathrm{~cm}^{2}\right)$. Each Silicon is a $500 \mu \mathrm{m}$ thick detector of dimension $7 \times 5 \mathrm{~cm}$. The detectors are mounted on a glass epoxy board in a close packed geometry minimizing dead gaps. Each detector is directly connected to its charge sensitive pre-amplifier mounted on the boards. The whole detector array is mounted inside the same focal plane chamber box housing the ionization chamber electrodes avoiding the exit window for the IC.

\subsubsection{Signal readout electronics}

The signal readout for the 128 pads in each drift chamber is based on 4 ASIC GASSIPLEX chips, each of which has 32 analog input channels and one multiplexed analog output. Each analog channel consists of one charge sensitive low noise pre-amplifier, a shaping amplifier and a track and hold stage. The track and hold signal is provided by the wire signal. For each channel the collected charge is converted into voltage which is multiplexed in the output and fed to a fast analog to digital converter(ADC)(CAEN CRAM system). To cover the full dynamic range, the shaping time and gain of the channel is set to $0.5 \mu s$ and $12 \mathrm{mV} / \mathrm{fC}$ respectively. The readout system for the two drift chambers consists of 4 CAEN CRAMS modules and two CRAM sequencers (CAEN V551B). Each C-RAM module has two ADC inputs each of which converts the output of 32 analog channels within a conversion time of $16 \mu \mathrm{s}$. For data acquisition, the master event trigger is provided by the fast timing signal from the SED detector or in some cases from the Silicon detector. The analog signals from other detectors are processed through standard NIM electronics consisting of pre-amplifiers, amplifiers and discriminators. The signals are digitized by ADCs and collected event-by-event using the VME based standard data acquisition system [12] at GANIL.

\section{Event Reconstruction and Particle Identification}

\subsection{Trajectory reconstruction}

Trajectory reconstruction in magnetic spectrometers have been used to correct for higher order aberrations and thereby improve the momentum resolution $[13,14]$. Spectrometers employing such techniques are often called as "software" spectrometers requiring the particle momentum and scattering angles to be determined by software reconstruction from the measured coordinates on the focal plane. The technique involves an accurate measurement of final position and angle, and retroactively reconstructs the full trajectory of the particles through the electric and magnetic fields using software algorithms. Generally such algorithms use complex mathematical transformation ma- trices to map the measured coordinates at the focal plane back to the target. The transformation matrix depends on the optical properties of the spectrometer and also require a precise knowledge of the transport of particles through the magnetic system. Two different approaches have been used in literature for the reconstruction algorithm. One is based on the computation of the inverse transfer map of an arbitrary order using COSY INFINITY [15] while the other is based on a numerical method using ray-tracing and polynomial parametrization (MOTER) [16]. In the case of VAMOS, we use a numerical method similar to MOTER but with a different approach for the polynomial parametrization. The technique uses extensive ray-tracing of particles through the spectrometer, mapping the full acceptance phase space and building a database of these particle coordinates. The reverse mapping of the coordinates is obtained by expressing the nonlinear map as a polynomial function. To generate the database for particle coordinates, particle trajectories are simulated through the magnetic field system using the ion optical ray-tracing code ZGOUBI [7]. This is achieved as follows.

To determine the trajectory of a particle along the spectrometer, we use the standard ion optics formalism [17] in which each particle state is characterized by a six parameter vector $(x, \theta, y, \phi, l, \delta)$ described relative to a reference trajectory whose momentum $p_{0}$ is used to set the magnetic field strength. For a given arbitrary trajectory, the parameters $x$ and $y$ correspond to two transverse distances from the reference trajectory (see Fig. 3), $\theta$ is the angle between the $z$-axis and the projection of the velocity vector of the trajectory on the $x z$ plane (symmetry plane), $\phi$ refers to the angle between the velocity vector and its projection on the $x z$ plane, $\delta=\left(p-p_{0}\right) / p_{0}$ defines the fractional momentum deviation from the reference momentum and $l$ the difference in path length between the given and the reference trajectory. For ray-tracing purposes, a set of 20000 particles is prepared in the initial phase space starting at the origin $x, y=0$ (considering a well focused beam, the initial coordinates are set zero) with well defined angle $(\theta, \phi)$ and momentum deviation $(\delta)$. These were generated by binning the initial phase space parameters $\theta, \phi, \delta$ into 20000 vectors each corresponding to a trajectory defined by unique $(\theta, \phi, \delta)$ values. These vectors fill a maximum angular and momentum acceptance of the spectrometer defined within the range $\Delta \theta, \Delta \phi= \pm 160 \mathrm{mrad}$ and $\delta= \pm 10 \%$. The trajectories of the particles were simulated through VAMOS electromagnetic model using code ZGOUBI. Realistic field descriptions for each magnetic element are considered in the calculation by incorporating the $3 \mathrm{D}$ field maps generated using electromagnetic computation code TOSCA [18]. The code ZGOUBI traces a particle through a system of magnetic fields and calculates the final coordinates numerically by integrating the equation of motion in a magnetic field. The final coordinates of the trajectories are calculated on the "image plane" chosen between the two drift chambers and located $760 \mathrm{~cm}$ from the target. For each trajectory, its initial input parameters $\left(\theta_{i}, \phi_{i}, \delta\right)$ and its fi- 
nal calculated parameters $\left(x_{f}, \theta_{f}, y_{f}, \phi_{f}, l\right)$, are stored in an output file. The subscript ' $i$ ' and ' $f^{\prime}$ here refers to the parameters corresponding to initial and final phase space respectively. From the set of data generated from 20000 trajectories, the reverse map is determined numerically using a fitting procedure. This procedure computes the transformation of coordinates by expressing the initial parameters as a multivariate polynomial function of the four final parameters $\left(x_{f}, \theta_{f}, y_{f}, \phi_{f}\right)$ as outlined below.

For each transmitted trajectory the parameters that are to be reconstructed are expressed as a set of four independent non-linear functions:

$$
\begin{aligned}
& \delta=F_{1}\left(x_{f}, \theta_{f}, y_{f}, \phi_{f}\right) \\
& \theta_{i}=F_{2}\left(x_{f}, \theta_{f}, y_{f}, \phi_{f}\right) \\
& \phi_{i}=F_{3}\left(x_{f}, \theta_{f}, y_{f}, \phi_{f}\right) \\
& l=F_{4}\left(x_{f}, \theta_{f}, y_{f}, \phi_{f}\right)
\end{aligned}
$$

The four non-linear transfer functions ' $F$ ' in the above equations is expressed as a $7^{\text {th }}$ order polynomial of four variables $\left(x_{f}, \theta_{f}, y_{f}, \phi_{f}\right)$. It has been found that the inclusion of higher order coefficients did not significantly improve the resolution.

As an example, the expression for $\delta$ reads:

$$
\delta=\sum_{i, j, k, l=0}^{i+j+k+l=7} C_{i j k l} x_{f}^{i} \theta_{f}^{j} y_{f}^{k} \phi_{f}^{l}
$$

where the coefficients $C_{i j k l}$ are related to the properties of the inverse transfer map of the system. Due to mid plane symmetry in the system, the coefficients in Eq. 2 are null for odd values of $k+l$ and only the remaining relevant 150 coefficients are considered for reconstructing the $\delta$ parameter. Writing Eq. 2 for each trajectory define a set of linear equations corresponding to data points spanning the full space space. The unknown coefficients $C_{i j k l}$ are determined numerically. A program was developed for this purpose which determines the best converged solution by fitting the polynomial expression to the set of trajectories computed by ZGOUBI in an iterative procedure. A similar procedure is applied to the other three parameters $\theta_{i}, \phi_{i}$ and $l$, extracting the full set of coefficients corresponding to each reconstructed parameter. It is to be noted that for the parameter $\phi_{i}$, the coefficients are null when $k+l$ is even. Once the $C_{i j k l}$ coefficients are determined, they are passed to the reconstruction algorithm in order to map the measured final coordinates data on an event by event basis. Since the algorithm is independent of any optics code once the coefficients are fixed, it can easily be adopted in both on-line and offline event identification. To further improve upon the existing method, an alternate reconstruction procedure using piecewise polynomials of lower order has also been tested [19]. a)

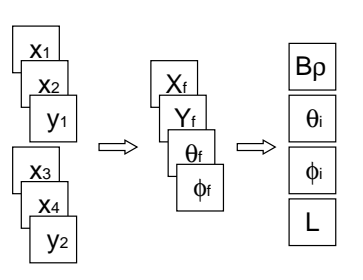

b)

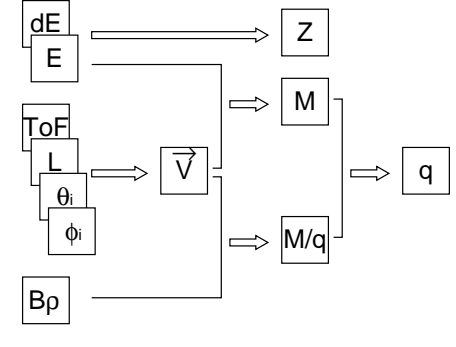

Figure 5. Flow chart showing the identification algorithm. Part a) shows the reconstruction from measured $x, y$ positions from the two drift chambers. Each drift chamber gives two values of $x$, from the two segments of PADs, and one $y$ position measurement. Part b) shows the steps along the identification procedure correlating measured quantities and reconstructed parameters.

\subsection{Identification}

For a complete identification of the reaction products, the following basic relation between $B \rho$, the measured quantities $(E, T O F)$ and particle characteristics ( mass number $M$, velocity $v$ and atomic charge state $q$ ) are used.

$$
\begin{aligned}
\frac{M}{q} & =\frac{B \rho}{3.105 \times \beta} \\
M & =\frac{2 E_{t o t}}{931.5 \times \beta^{2}}
\end{aligned}
$$

where $E_{\text {tot }}$ is the total energy in $\mathrm{MeV}, B \rho$ in $\mathrm{T}-\mathrm{m}$, and $\beta=v / c$. An event by event particle identification is thus obtained through the following steps.

a) Determine the four parameters $x_{f}, \theta_{f}, y_{f}, \phi_{f}$ from measurements by the two drift chambers.

b) Reconstruct $B \rho$, the initial angles $\theta_{i}, \phi_{i}$ and the path length $l$ from the above four parameters.

c) Determine the velocity $v$ from the measured TOF and reconstructed $l$, using $v=\frac{l}{T O F}$.

d) Determine $\frac{M}{q}$ and $\operatorname{Mass}(M)$ using Eq. 3 .

e) Determine the ionic charge $q$ from $M$ and $\frac{M}{q}$.

f) Identify the atomic number $Z$ based on the measurement of the energy loss $\Delta E$ and energy $E$.

A flow chart of the above procedure is shown in Fig. 5. It is implemented as a standard $\mathrm{C}++/$ FORTRAN subroutines in data analysis code.

\subsection{Applications to the identification of products formed in deep inelastic transfer reactions}

In the following part we show the results of the trajectory reconstruction and identification method applied to the data of a recent measurement [6], demonstrating the unique capability of the VAMOS spectrometer for identifying weak reaction channels. The aim of the experiment was to study the evolution of shell structure in neutron rich nuclei around $N=34$ produced in deep inelastic reactions. The experimental setup consisted of the VAMOS spectrometer coupled to the high efficiency $\gamma$-detector ar- 


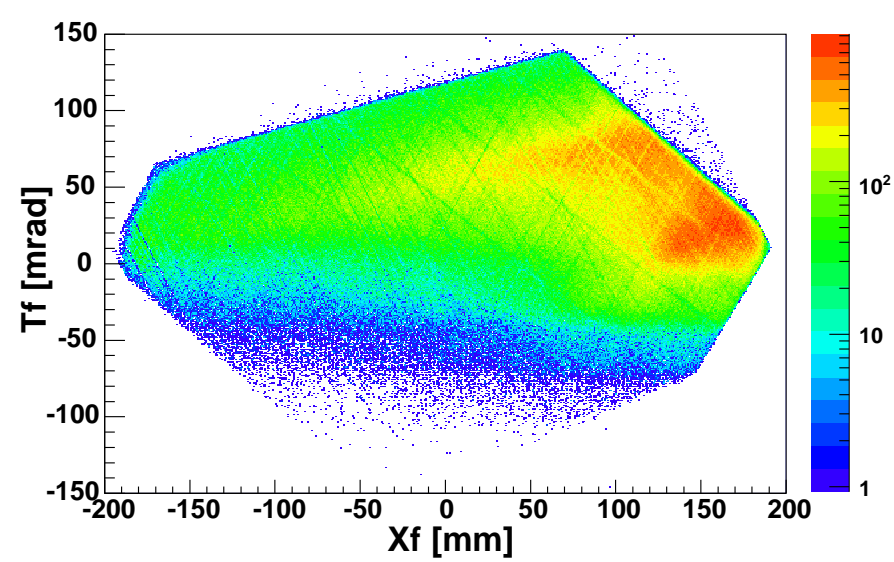

Figure 6. The raw image at the "image plane" showing the angle $\theta_{f}$ plotted as a function of position $\mathrm{x}_{f}$ obtained from the drift chambers in the focal plane.

ray EXOGAM allowing the measurement of coincident $\gamma$ rays of the residues.

The experiment was performed with a ${ }^{238} \mathrm{U}$ beam at 1.31 $\mathrm{GeV}$ bombarding an isotopically enriched $1 \mathrm{mg} / \mathrm{cm}^{2}$ thick ${ }^{48} \mathrm{Ca}$ target. Target like recoils resulting from deep inelastic transfer were detected and identified by VAMOS. The spectrometer was rotated to $35^{\circ}$ (around the calculated grazing angle) with respect to the beam direction. The primary beam with an intensity of $2 \mathrm{pnA}$ (particle nanoampere) current after striking the target was stopped in the Faraday cup at zero degree behind the first quadrupole. The spectrometer was operated in the momentum dispersive mode with maximum acceptance covering reaction products emitted within the range $\Theta_{l a b}=28^{\circ}$ to $41^{\circ}$. After a series of $B \rho$ scans, the magnetic field were set for selecting mass $M=52$ with charge state $q=19^{+}$and energy $E=430 \mathrm{MeV}$ optimizing the detection of ${ }^{52} \mathrm{Ca}$ like products at the image plane. The products were detected using the standard detection system (see Fig. 3) as described before. The time of flight was recorded between the beam frequency and the SED as well as between SED and the silicon array. Thus the reaction products were characterized by an event by event measurement of $x, y, \theta, \phi, \triangle E, E$ and $T O F$. Signals from all the silicon detectors and ionization chamber pads were initially gain matched in energy and time using a pulse generator and in-beam calibrations using both the direct beam and elastic recoils. Timing signals from the SED detector were used as the master event trigger. All detector signals were processed using standard NIM and VME electronics and were collected event-by-event using the GANIL data acquisition system which was set up to run in master-slave configuration recording both VAMOS and EXOGAM events within a common dead-time [12].

The trajectory parameters $x_{f}, y_{f}, \theta_{f}, \phi_{f}$ of each detected particle were determined by projecting the data from the drift chambers on the image plane. Fig. 6 shows the resulting image displaying the angle $\theta_{f}$ plotted as a function of the position coordinate $x_{f}$. The reconstruction procedure is then applied to the data in order to determine the magnetic

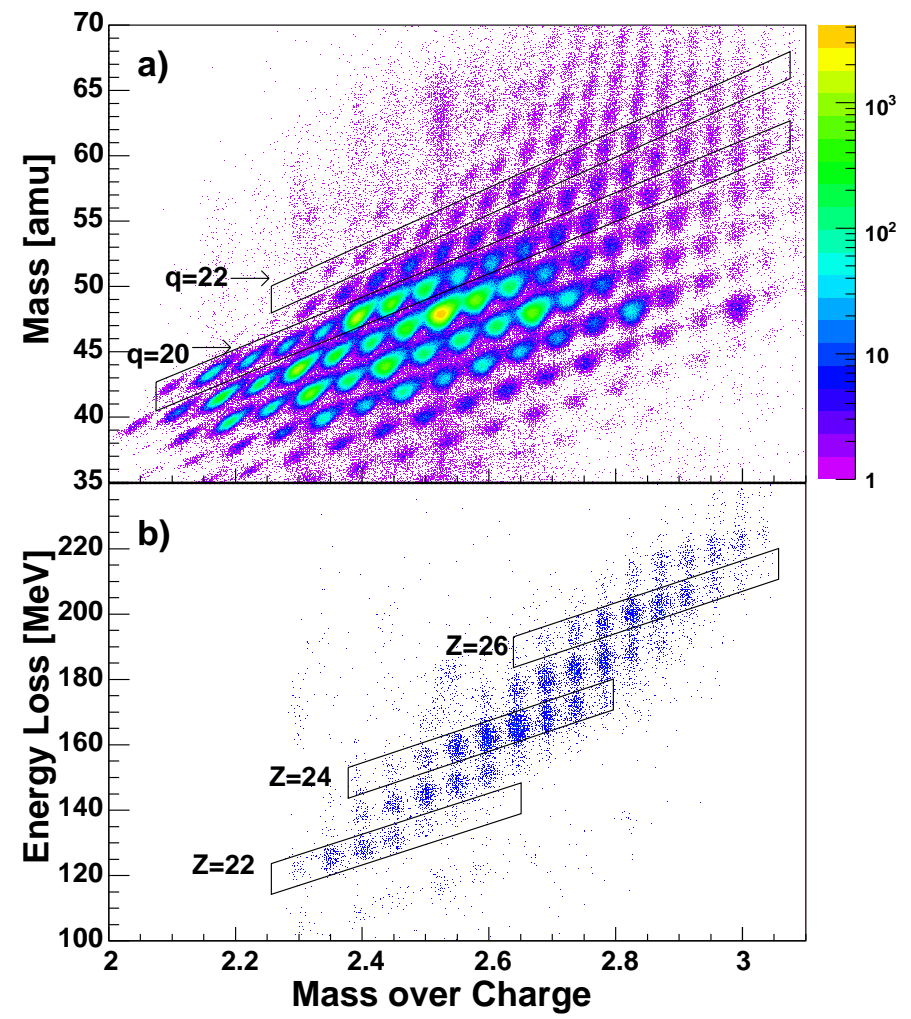

Figure 7. a) Spectrum showing correlation between mass $(M)$ and mass over charge $(M / q)$. The data shown here correspond to one Silicon detector. b) Identification plot of $\Delta E$ plotted as a function of $M / q$ showing unambiguous identification of products. The spectrum shown here is generated by gating on one particular charge state $q=22$.

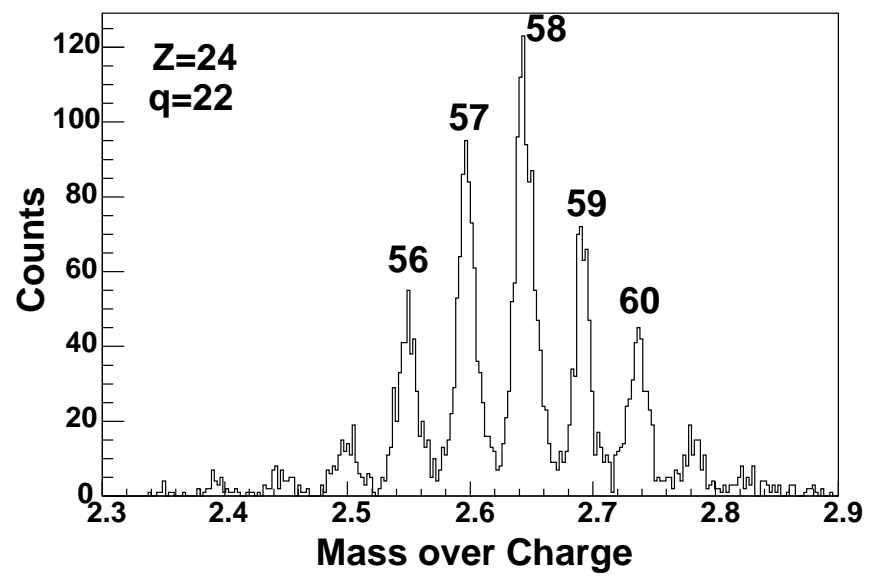

Figure 8. Mass spectrum for the Chromium element $(Z=24)$. The FWHM corresponds to a mass resolution of $1 / 165$.

rigidity $B \rho$, path length $l$ and initial angles $\left(\theta_{i}, \phi_{i}\right)$ of the reaction products on an event by event basis. Unique identification in terms of $M$ and $Z$ is finally achieved according to the scheme of Fig. 5. The resulting identification spectra from this experiment is shown in Fig. 7. Figure 7a shows a two dimensional image of mass $M$ plotted as a function of mass over charge $(M / q)$. The bands clearly seen in the figure correspond to different charge states accepted by the spectrometer. The data shown here are those collected by 

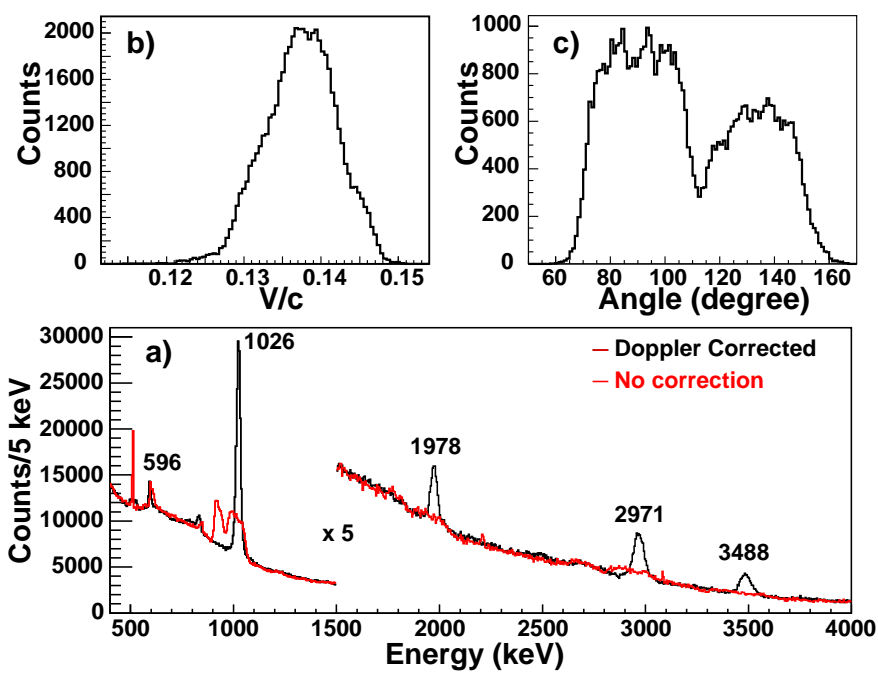

Figure 9. a) $\gamma$-ray energy spectrum in coincidence with ${ }^{50} \mathrm{Ca}$ selected and identified by VAMOS. The full (gray) line corresponds to Doppler corrected (uncorrected) spectrum. b) The measured velocity distribution in VAMOS of the ${ }^{50} \mathrm{Ca}$ fragments. c) The yield of the gamma rays from the ${ }^{50} \mathrm{Ca}$ fragments as a function of angle between the detected gamma ray and the ${ }^{50} \mathrm{Ca}$ ion.

one silicon detector covering only a small region of the image plane. Similar results were obtained for using the other Si detectors. Masses belonging to different nuclei are seen to be well separated making distinct identification possible. By selecting a software gate on a particular charge state and combining the energy loss $\Delta E$ signal with the mass over charge $(M / q)$, masses corresponding to particular elements are identified as shown in Fig. 7b. The spectrum in Fig. $7 \mathrm{~b}$ is generated by putting a gate on $q=22$ showing $(Z, A)$ identification of target like residues with $Z \geq 22$. By projecting the data selected for a particular $Z$, the corresponding mass spectrum can be obtained. One example of such a mass spectrum for the Chromium isotopes is shown in Fig. 8. The mass resolution achieved in this experiment was $\frac{\Delta M}{M} \sim \frac{1}{165}$. It was limited mainly by the accuracy of the $T O F$ measurement. All these measurements were done with a $T O F$ resolution of $\sim 1$ ns mainly limited by the beam frequency $(\mathrm{RF})$.

The coincident prompt $\gamma$ rays corresponding to identified fragments were detected using the EXOGAM detector array consisting of 11 segmented clover detectors in a close packed geometry at $11.4 \mathrm{~cm}$ from the target. Eight detectors were placed at $90^{\circ}$ and three at $135^{\circ}$ with respect to the central angle in VAMOS. Each electrical segment of a clover detector subtended an angle of $\sim 5.1^{\circ}$. The partial Compton suppression as opposed to the full Compton suppression configuration (minimum distance $14.7 \mathrm{~cm}$ ) was preferred so as to maximize the efficiency. The total efficiency of the 11 clover array has been measured to be $\sim$ $9 \%$ at $1.33 \mathrm{MeV}$ using addback mode (summing the energy signal from neighboring individual crystals firing in an event). Particle-gamma coincident data permitted to select the $\gamma$-rays belonging to one particular nucleus by setting appropriate gates on $Z$ and mass $M$. Fig. 9a shows the $\gamma$ - spectrum obtained in coincidence with ${ }^{50} \mathrm{Ca}$ selected and identified in VAMOS as explained earlier. $\gamma$-rays belonging to other neutron rich nuclei, ranging from $\mathrm{Ar}$ to $\mathrm{Fe}$, have also been identified for the first time in the present experiment and will be reported elsewhere. It should be noted that, despite the high velocity of the recoils $(\beta \sim 0.14)$, the finally achieved resolution of the gamma rays energy crucially relies on the event by event Doppler correction using the reconstructed velocity and emission angles of the particle as given by VAMOS. Measurements of the level scheme of ${ }^{48} \mathrm{Ar}$, where the only earlier information was its halflive, were also possible due to this unique combination of the EXOGAM and VAMOS. These results illustrates that significant enhancement in selectivity can be achieved by coupling VAMOS and EXOGAM together. Further details of some of the experimental results can be found in Ref. [6].

\section{Acceptance of VAMOS}

The transmission of reaction products through the spectrometer depends on both, the reaction kinematics and the maximum acceptance of the spectrometer. The reaction kinematics define the angle and momentum distribution of the particles entering the spectrometer whereas the acceptance put the limit on the range of angle and momentum that can be transmitted by the spectrometer without loss. For a fixed setting of the magnet, the spectrometer transmits particles within a limited range of angle and momentum around the central value. The spectrometer acceptance (accepted momentum and angular range) depends on the ion optical features and the mechanical constraints such as magnetic apertures, detector size, slits and vacuum pipes in the system. Ion optical calculation shows that, the transmission of particles through VAMOS is non-uniform across its accepted range of angles and momentum. In this section we discuss the results of the Monte Carlo simulations performed to estimate the acceptance of the spectrometer and its application to data from a quasi elastic scattering experiment at an energy near the Coulomb barrier.

We have performed a comprehensive study of the transmission of particles through VAMOS by simulating the trajectory of the particles inside spectrometer using the ZGOUBI code. The input to the code contained the description of ion optical elements, field maps, positioning of mechanical apertures, detector configuration and a set of incoming particles. The latter have been chosen to have a uniform distribution within the initial $\theta, \phi, \delta$ phase space. More than 600,000 trajectories were sampled within an input range of $\Delta \theta, \Delta \phi= \pm 200 \mathrm{mrad}$ (exceeding the angular aperture of the spectrometer) and $\delta= \pm 20 \%$. The calculation was performed with existing detector size that limits the trajectories. The analysis of the trajectory computed by ZGOUBI determines whether a particle characterized by a particular combination of $\theta, \phi, \delta$ reaches the detectors or, it is lost in transport. For a given setting of the spectrometer, the ratio of the number of particles reaching the detec- 

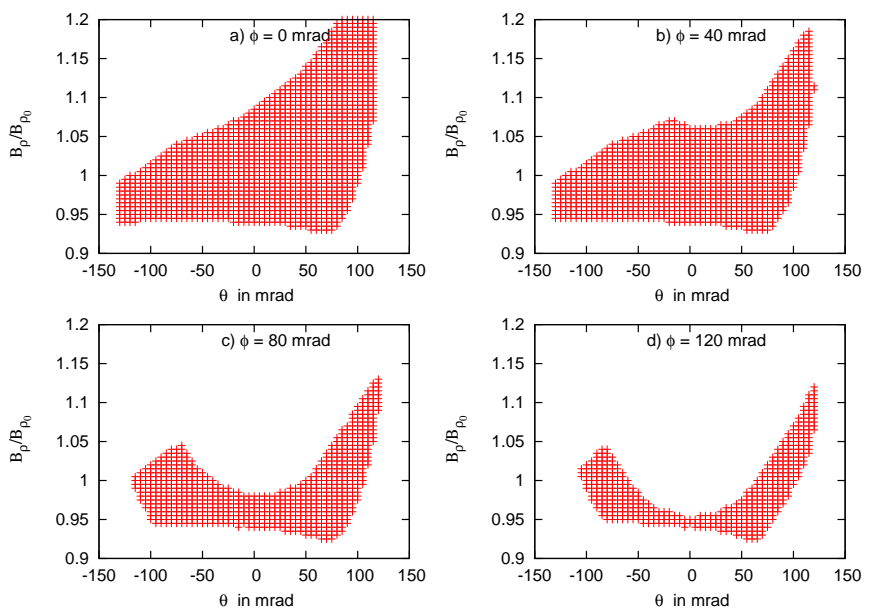

Figure 10. Relative rigidity plotted as a function of angle $\theta$ (horizontal plane) at various vertical angle $\phi$.

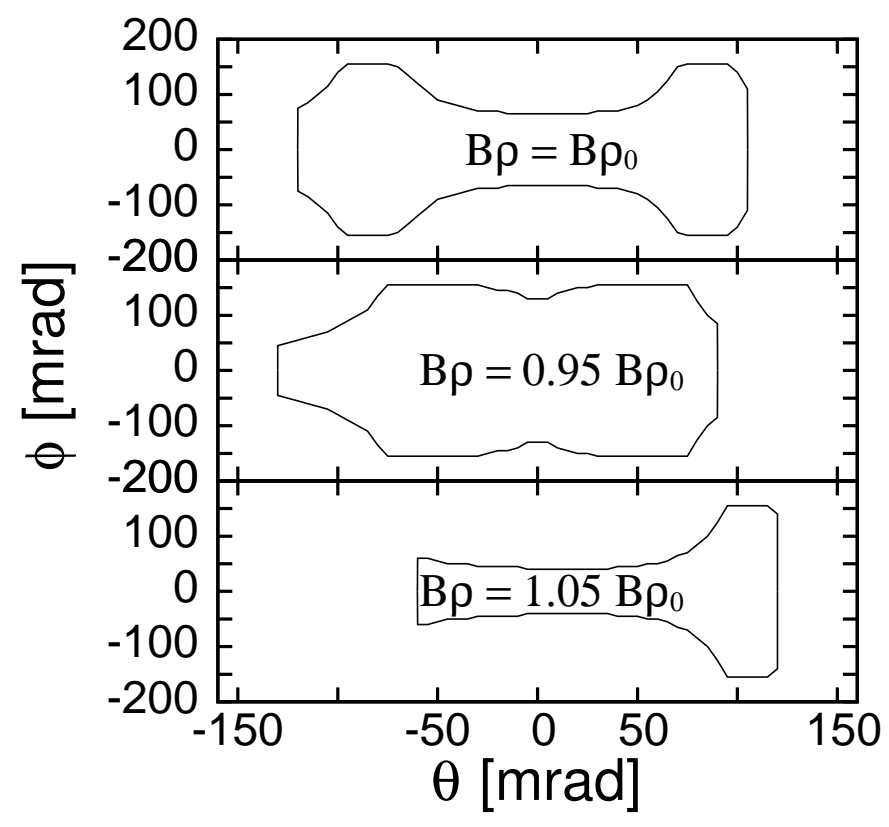

Figure 11. Range of angles accepted in vertical plane as a function of horizontal angle. The top panel corresponds to particles with reference rigidity $B \rho=B \rho_{0}$, the middle with $B \rho=0.95 B \rho_{0}$ and the bottom with $B \rho=1.05 B \rho_{0}$.

tor to the number of incoming particles determines the total transmission through the spectrometer. Recording the rigidity and initial angles for each transmitted particle, an acceptance map was generated as displayed in Fig. 10. The figure shows the accepted range of relative rigidity plotted as a function of the angle accepted in horizontal plane. Fig. 10a-d shows the variation of the acceptance across the vertical plane (corresponding to different $\phi$ ) of the spectrometer. The arbitrary shape of the two dimensional surface ( which is a measure of the acceptance) and its variation across the vertical plane implies that, the transmission is non-uniform and vary strongly as a function of rigidity and angle.

In Fig. 11, the same data is shown plotted with the range

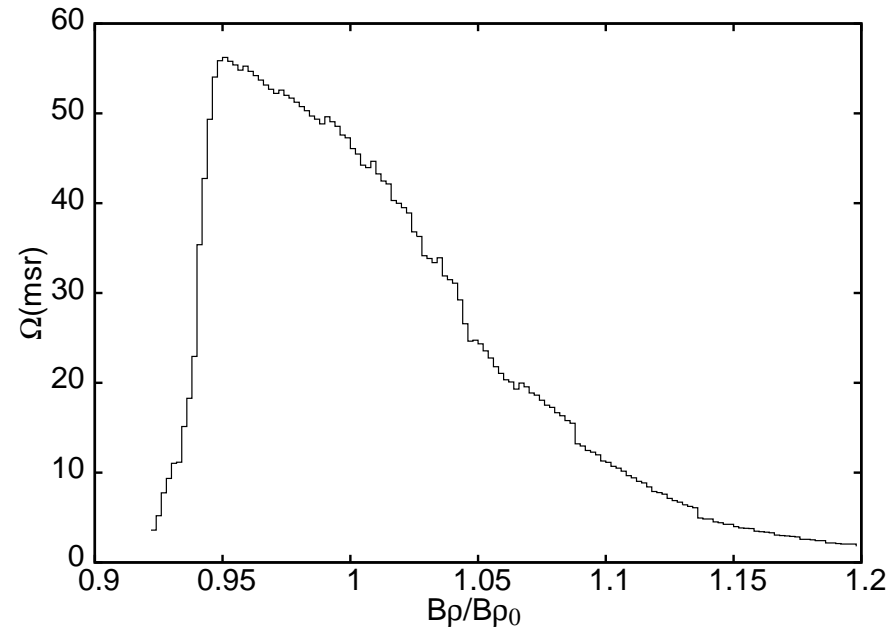

Figure 12. Effective solid angle(msr) of VAMOS as a function of relative rigidity. The solid angle is obtained by integrating over the range of accepted angles $\theta$ and $\phi$.

of vertical angles as a function of the horizontal angle for three different rigidity values $\left(B \rho=B \rho_{0}, B \rho=0.95 B \rho_{0}\right.$ and $B \rho=1.05 B \rho_{0}$ ). It shows the effective solid angle (the area integrated over the range of input angles) varying nonuniformly as a function of rigidity. It is evident that, for any reaction measurement using VAMOS, the measured counts would be influenced by the variation in effective solid angle and the results needs to be corrected accordingly. Since there is no functional formula describing the dependence of solid angle as a function of rigidity, the experimental data needs to be sorted and corrected on event-by-event basis taking into account the solid angle limits. This could be achieved by binning the data into finite steps of $B \rho$ and $\theta$, and correcting the effective solid angle for each bin on an event-by-event basis. The effective solid angle for a given magnetic rigidity is determined from the limits of horizontal and vertical angles accepted by the spectrometer using the relation

$\Delta \Omega=\int_{\theta 1}^{\theta 2} \sin \theta d \theta \int_{\phi 1}^{\phi 2} d \phi$

where the integration is performed over the limits of $\theta$ angular bin size and the $\phi$ acceptance limit. By repeating the same procedure for all accepted rigidities, an effective solid angle curve has been built, the result of which is shown in Fig 12. The figure shows how the effective solid angle (integrated over the accepted range of angles) for VAMOS varies as a function of relative rigidity of the particle.

To illustrate the effect of transmission loss on reaction measurement, a computer program was developed to simulate the angular distribution of particles in laboratory system. The simulation utilizes particles with an isotropic distribution of angles, select randomly and trace them through optical configuration of spectrometer set at $35^{\circ}$. For each particles reaching the image plane, its rigidity and initial angles were recorded and an intensity pattern was built. The resulting angular distribution is displayed in Fig. 13, 


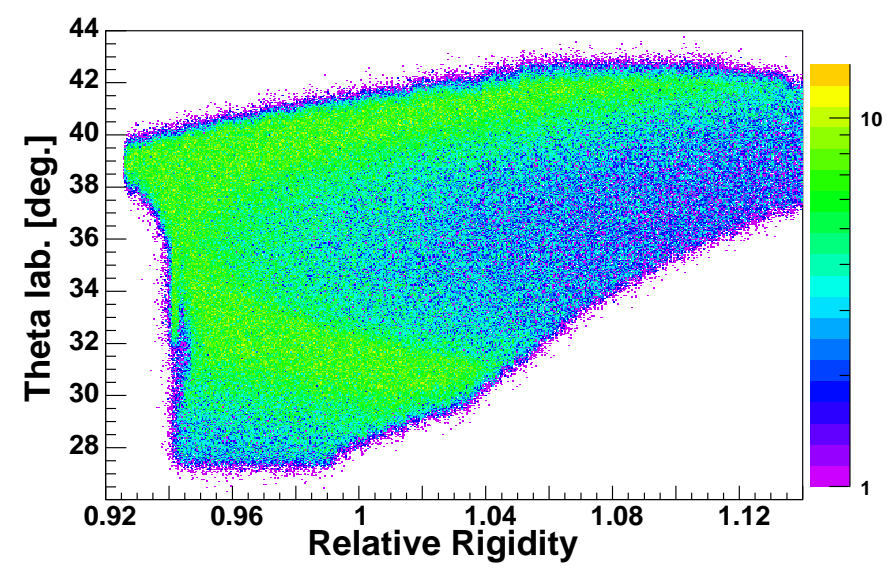

Figure 13. Angular distribution (simulation) of particles reconstructed after transmission through VAMOS. The isotropic distribution of incoming particles folded by transmission loss in the spectrometer, results in a non isotropic distribution.

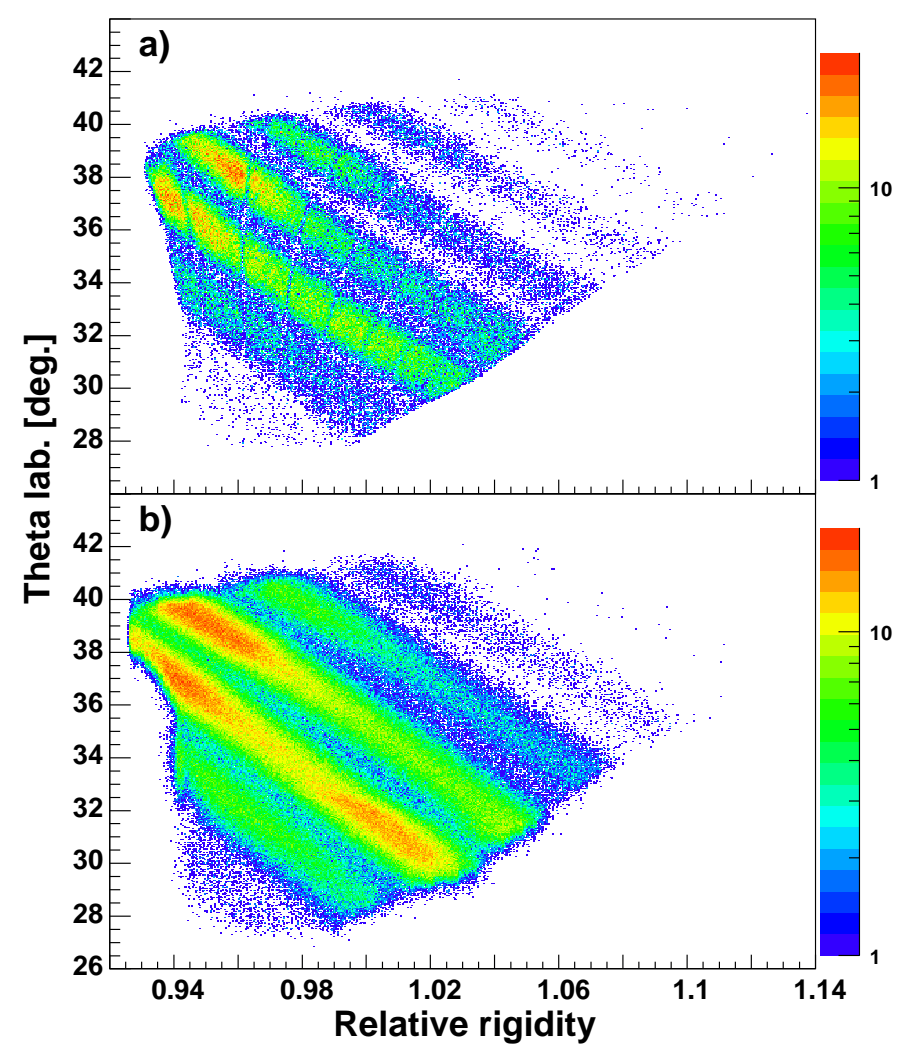

Figure 14. Two dimensional plot showing $\theta_{L a b}$ plotted as a function of $B \rho$. a) Experimental data corresponding to scattered ${ }^{58} \mathrm{Ni}$ target recoils. The various bands correspond to different charge states of the products accepted in a single setting of VAMOS. b) Result of the simulation taking into account the acceptance cut of spectrometer, see the text. A Gaussian distribution of charge state is considered in simulation.

which shows non-isotropic distribution due to intensity loss of particles in spectrometer.

To check the validity of this calculation, this method was applied to test the data from a recent elastic scattering experiment. The experiment was performed using a ${ }^{238} \mathrm{U}$ beam at $1.31 \mathrm{GeV}$ impinging on a $1 \mathrm{mg} / \mathrm{cm}^{2}{ }^{58} \mathrm{Ni}$ target. The spectrometer was rotated by $35^{\circ}$ with respect to beam direction, and set for selecting elastic ${ }^{58} \mathrm{Ni}$ recoils with charge state $q=26$. For each detected particle, the magnetic rigidity and scattering angle were determined according to the reconstruction method detailed in section 4 . Fig. 14a shows the measured angular distribution of the ${ }^{58} \mathrm{Ni}$ recoils plotted as a function of relative rigidity. In one setting of the spectrometer, particles scattered in the angular range from $\Theta_{l a b}=28^{\circ}$ to $\Theta_{l a b}=41^{\circ}$ are accepted by the spectrometer. As can be seen in the figure, the angular distribution shows fragmented into many bands corresponding to various populated charge states. The spectrum shows seven charge states of the recoils accepted by VAMOS. The narrow gaps ( seen along the $\mathrm{Y}$ axis) in the experimental data corresponds to the missing counts due to the gaps in silicon detector arrays. In Fig 14b, similar results from the Monte Carlo simulation is displayed. The simulation program consisted two parts: the kinematics for elastic scattering and the application of VAMOS acceptance cuts to the data. Using scattering kinematics, the program determine the scattering angle and magnetic rigidity of the particles, and distributes them in spectrometer coordinates $\theta, \phi, B \rho$. The program assumed a Gaussian distribution of charge states for the particles. For each simulated particle, its $B \rho, \theta, \phi$ values are compared with the limits of VAMOS acceptance and an event is accepted if it falls within the acceptance limit of the spectrometer. The angular distribution of the accepted events is shown in Fig $14 \mathrm{~b}$. The results of the simulation also showed intensity distribution with different bands of charge states similar to the experimental results. The simulations did not include the detailed geometry of the Si detectors. The close agreement between the simulation and the measurement gives us the confidence in using the calculated acceptance map for correcting experimental data. By using experimentally measured charge state distribution and applying correction to the data to take care of the intensity loss, the spectrometer can be used for reaction measurement.

\subsection{Effect of Detector size on Acceptance.}

A detailed investigation of the acceptance property of VAMOS has shown that, the present detectors with a horizontal size of $40 \mathrm{~cm}$ is causing sharp discontinuity on the lower part of the $B \rho$ acceptance (See Fig 12). A significant improvement in $\mathrm{B} \rho$ and $\theta$ acceptance can be achieved by doubling the size of the detectors along the dispersive direction. Particles having lower rigidity entering the spectrometer with positive angle $(\theta)$ will be counted by larger size detector which otherwise would have been lost as is the case with the present setup. Calculation also showed that, not much gain to be achieved on the vertical side, as the vertical acceptance already reached the maximum limit set by the dipole gap. With these observations, it is proposed to build a set of larger detectors in the future. 


\section{Conclusions}

We have presented the performance of the wide acceptance VAMOS spectrometer at GANIL. A numerical method for trajectory reconstruction has been developed and applied successfully to identify reaction products. The spectrometer acceptance has been studied by simulating the trajectory of the particle inside the magnets using an ion optical code. The large acceptance with ray-tracing capability and good identification properties make the spectrometer a versatile instrument for selecting exotic reaction channels in nuclear reactions. VAMOS coupled with EXOGAM detector array has been used for the investigation of spectroscopy of neutron rich nuclei produced in deep inelastic reactions. Using reconstructed velocity and angles of the recoiling nucleus, Doppler correction of gamma rays of individual nuclei could be applied enhancing the detection sensitivity of the setup.

\section{Acknowledgment}

The authors would like to thank all their colleagues who have contributed at various stages of this work. They also acknowledge the strong support of the GANIL technical staff towards the smooth running of the various facilities. Part of this work was funded by the European Commission under contract 506065 (EURONS/INTAG) and one of us (S. P) also acknowledges the above for full financial support during his stay at GANIL.

\section{References}

[1] H. Savajols and VAMOS collaboration, Nucl. Phys. A654 (1999) $1027 \mathrm{c}$.

[2] A.M. Stefanini et al., Nucl. Phys. A701 (2002) 217c.

[3] A. Cunsolo et al., Nucl. Instr. and Meth. A481 (2002) 48.

[4] A.C.C. Villari et al., Nucl. Phys. A 693 (2001) 465.

[5] J. Simpson et al., Heavy Ion Phys. 11 (2000) 159.

[6] M. Rejmund et al., Phys. Rev. C 76 (2007), 021304(R).

[7] F. Meot , Nucl. Instr. and Meth. A427 (1999) 353.

[8] E. Bougamont, DSM-DAPNIA report No: 6D6810E2100/303, 2002.

[9] J.C. Santiard et al., CERN-ALICE-PUB-2001-49.

[10] A. Drouart et al., Nucl.Instr.and Meth. A 579 (2007) 1090.

[11] A.N. James et al., Nucl.Instr.and Meth. 212 (1983) 545.

[12] B. Raine, M. Tripon and B. Piquet, IEEE Transactions on Nuclear Science, Vol 41, No 1 (1994) 55; http://hal.in2p3.fr/in2p3-0010517/fr/.

[13] H. Blok et.al., Nucl. Instr. and Meth. A 262 (1987) 291.

[14] M. Berz et.al, Phys. Rev. C 47 (1993) 537.

[15] M. Berz, Nucl. Instr. and Meth. A 298 (1990) 473.

[16] H. A. Thiessen and M. Klein, Proceedings of the Fourth International Conference on Magnet Technology, NTIS CONF720908-12, p. 8., 1972.

[17] Davids C. Carey, Optics of Charged Particle beams, Harwood Academic Publishers, 1987.

[18] TOSCA Static Field Analysis OPERA-3D, Vector Fields, 24 Bankside Kidlington, Oxford, England.

[19] S. Pullanhiotan, A. Chatterjee, B. Jacquot, A. Navin and M. Rejmund, Proceedings of $X V^{t h}$ International Conference on
Electromagnetic Isotope Separators and Techniques related to their applications, EMIS2007, to be published in Nucl. Instr. and Meth. 